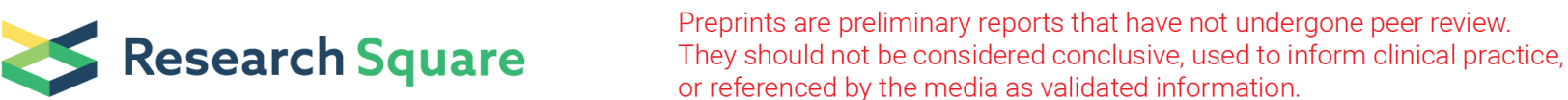

\section{Withanone from Withania somnifera May Inhibit Novel Coronavirus (COVID-19) Entry by Disrupting Interactions between Viral S-Protein Receptor Binding Domain and Host ACE2 Receptor}

\section{Acharya Balkrishna}

Patanjali Research Foundation Trust

\section{SUBARNA POKHREL}

Patanjali Research Foundation Trust

\section{Jagdeep Singh}

Patanjali Research Foundation Trust

Anurag Varshney ( $\nabla$ anurag@prft.co.in )

Patanjali Research Institute https://orcid.org/0000-0001-8509-0882

\section{Research}

Keywords: COVID-19, ACE2-RBD complex, Withania somnifera, Withanone, Docking, Molecular Dynamics simulation, Salt Bridge, electrostatic component of binding free energy

Posted Date: March 20th, 2020

DOI: https://doi.org/10.21203/rs.3.rs-17806/v1

License: (c) (i) This work is licensed under a Creative Commons Attribution 4.0 International License. Read Full License

Version of Record: A version of this preprint was published on November 20th, 2020. See the published version at https://doi.org/10.2147/DDDT.S292805. 


\section{Abstract}

\section{Background}

Newly emerged COVID-19 has been shown to engage the host cell ACE2 through its spike protein receptor binding domain (RBD). Here we show that natural phytochemical from a medicinal herb, Withania somnifera, have distinct effects on viral RBD and host ACE2 receptor complex.

\section{Methods}

We employed molecular docking to screen thousands of phytochemicals against the ACE2-RBD complex, performed molecular dynamics (MD) simulation, and estimated the electrostatic component of binding free energy, along with the computation of salt bridge electrostatics.

Results

We report that W. somnifera compound, Withanone, docked very well in the binding interface of AEC2RBD complex, and was found to move slightly towards the interface centre on simulation. Withanone significantly decreased electrostatic component of binding free energies of ACE2-RBD complex. Two salt bridges were also identified at the interface; incorporation of Withanone destabilized these salt bridges and decreased their occupancies. We postulate, such an interruption of electrostatic interactions between the RBD and ACE2 would block or weaken COVID-19 entry and its subsequent infectivity.

Conclusion

Our data, for the first time, show that natural phytochemicals could well be the viable options for controlling COVID-19 entry into host cells, and W. somnifera may be the first choice of herbs in these directions to curb the COVID-19 infectivity.

\section{Introduction}

In December 2019, a mysterious virus causing pneumonia was first reported in China [1], and it is now growing globally into a deadly disease. In the first week of January 2020, the Chinese Centre for Disease Control and Prevention (CCDCP) identified a novel coronavirus strain that has not been earlier identified in humans [2-4]. Coronaviruses are characterized as zoonotic which can transmit between animals and people [5]. These are large positive-strand RNA viruses [6], which can cause infection in a variety of avian as well as mammalian species. These can cause diseases related to the central nervous system, upper and lower respiratory and gastrointestinal tracts $[7,8]$. Till now, seven coronaviruses have been identified that can cause human diseases, four of these are mild viruses: OC43, 229E, HKU1 and NL63 [9]. Whereas, other three viruses can have more serious consequences in human, and these are SARS-CoV (causes Severe Acute Respiratory Syndrome) which appeared in November 2002 [10, 11], in China. Another outbreak of MERS-CoV (causes Middle East Respiratory Syndrome) emerged in 2012 in Saudi Arabia [12]. Now, Coronavirus COVID-19 has gradually crossed the wall of China, and has spread throughout the 
world. Stringent efforts are being placed globally to contain COVID-19 spread. However, epidemiological information and clinical features of illness caused by COVID-19 is still at infancy at present $[13,14]$.

In a viral infection, the viral entry in the host cell is a critical step that can be exploited for anti-viral therapy [15]. Coronaviruses get access into the target animal cells via binding to cell-surface-associated receptors, and its entry can be barred by targeting the viral receptor-binding site with neutralizing antibodies (nAbs). There are also certain small molecules (like RFI-641 and VP-14637) which inhibit the entry of several viruses including respiratory syncytial virus $[16,17]$. In the case of Coronavirus, the viral entry is mediated by the Receptor-Binding Domain (RBD) of its spike (S) glycoprotein, which binds to the host cell receptor Angiotensin-Converting Enzyme-2 (ACE2) [18, 19]. The coronavirus S-protein, a structural protein responsible for the crown-like shape of the viral particles, is $\sim 1200$ aa long S-protein belonging to class-I viral fusion proteins, and contributes to the cell receptor binding, tissue tropism and pathogenesis [20]. It contains several conserved domains and motifs, and the trimetric S-protein is processed at the S1/S2 cleavage site by host cell proteases. The protein is divided (cleavage, or priming) at a conserved sequence AYT $\downarrow M$ (located 10 aa downstream of SLLR-ST) into an N-terminal S1ectodomain that recognizes a cognate cell surface receptor and a C-terminal S2-membrane-anchored protein involved in viral entry [20-22]. The SARS-CoV S1-protein contains a conserved RBD, which recognizes the host ACE2. The RBD surface of S1/ACE2 implicates 14 aa in the S1 of SARS-CoV [23], among them, 8 residues are strictly conserved in COVID-19, supporting the hypothesis that ACE2 is also the receptor of this newly emerged coronavirus [24]. The RBD of COVID-19 differs largely from the SARS$\mathrm{CoV}$ at the C-terminus, and it has been reported that such difference did not result in drastic changes in its capability to engage ACE2 receptor [25]. Therefore, RBD has been an attractive target for the researchers to abrogate coronavirus infection. Reports suggested that certain human antibodies recognized RBD on the S1 domain and inhibited the viral infection by blocking the attachment of ACE2 [26, 27]. Three possible mechanisms have been proposed where COVID-19 infection can be abrogated by blocking the interaction of spike protein and ACE2 (Fig. 1), along with the strategy followed in the present work.

Withania somnifera (L.) Dunal (Solanaceae), commonly known as Ashwagandha, one of the most valued medicinal plants of the traditional Indian systems of medicines, is used in more than 100 formulations of Ayurveda, and is thought to be therapeutically equivalent to Ginseng [28]. W. somnifera has been used as antiviral herb for the treatment of genital disease caused by Herpes Simplex Virus among African tribes [29], and shown to anti-influenza properties [30]. The aim of present study is to check the antiviral potential of W. somnifera ingredients against COVID-19 by means of computational methods. The core rationale of this study is to inhibit or weaken the interactions between the receptor and RBD by using phytocompounds that could block or hamper viral entry into the host cells.

\section{Materials And Methods}

\section{Structures}


We screened thousands of phytocompounds by in-silico method. The strategy was to find phytocompounds that bind at the protein complex interface, and perturb their interactions. In this regard, we observed that the phytocompounds from W. somnifera could fit into our strategy. For W. somnifera root extracts, Chaurasiya et al. [31] have utilized a reversed-phase HPLC method for the simultaneous analysis of nine structurally similar withanolides: 27-hydroxy withanone, 17-hydroxy withaferin A, 17hydroxy-27-deoxy withaferin $A$, withaferin $A$, withanolide $D, 27$-hydroxy withanolide $B$, withanolide $A$, withanone and 27-deoxywithaferin A. Our lab data (unpublished results) also showed significant concentration of Withanolide A, Withanolide B, Withaferin A and Withanone in W. somnifera sapling (1520 days old). Therefore, we studied these compounds in detail after preliminary screening. The 3D structures of all the phytocompounds were sourced from PubChem database (https://pubchem.ncbi.nlm.nih.gov).

\section{Molecular docking}

The structure of the ACE2 complexed with spike protein receptor binding domain (RBD) of 2019-nCoV is not yet available. Therefore, RBD sequence of 2019-nCoV spike protein was obtained from NCBI (NCBI accession: QHD43416), and the mutations were considered [32]. The protein complex model was built using the MODELLER program as implemented in Structuropedia (http://structuropedia.org) [33] and by using SWISS-Model (http://swissmodel.expasy.org) based on a clean 3D0G (2.8 ^̊ resolution; Protein Data Bank, http://www.rcsb.org) template. The modelled 2019-nCoV RBD complexed with ACE2 A-chain was used for further processing after editing on PyMol [34]. Energy minimization was performed by 100 steps of steepest descent, followed by 500 steps of conjugate gradient using UCSF Chimera-1.13.1 [35], and the stereo-chemical quality of the energy minimized model was checked using VERIFY 3D [36], ERRAT [37], PROCHECK [38] and RAMPAGE (for Ramachandran plot) [39].

The SDF files of all the ligands downloaded from the PubChem database were then converted into PDB files using OpenBabel 2.4.1 [40]. The binding site in the ACE2-RBD complex was determined by blind docking using AutoDock Vina-1.1.2 (ADV-1.1.2) [41], and then the focused/targeted docking was performed based on the binding location of the ligands derived post blind docking. For intermediary steps, such as PDBQT files for protein and ligands preparation and grid box creation were performed using Graphical User Interface program AutoDock Tools-1.5.6 (ADT-1.5.6) [42]. ADT was used to assign polar hydrogens and Gasteiger charges. 'Choose ligand' option was used to set map file types. AutoDock Tool was used to save the prepared files in PDBQT format. For blind docking, Grid maps were prepared using a grid box size of $60 \times 60 \times 60 x y z$ points and the protein centre $(x=50.541, y=-1.366, z=105.966)$. To obtain the maximum number of poses, we set num_modes to 20 , energy range to 9 , and exhaustiveness to 8 . The pose with lowest energy of binding was extracted and aligned with the receptor for further analysis by Discovery Studio 2017 R2 Client [43] and PyMol[34]. In focused docking for Withanone, the grid was defined using AutoDock Tools 4 (ADT4) [42] to involve the binding location in the ACE2-RBD complex, by rendering side chains of the residues K8, L11, E12, N15, Y16, Q19, A369, Q370, P371, F372, R375 (ACE2 molecule) and R73, D75, K87, Y175 (RBD) as flexible. The size of the grid was set to $30 \times 30 \times 30 \mathrm{xyz}$ points with the grid centre of $32.575,-12.096$ and 110.756 . Docking simulations 
were initiated with the random seed. Discovery Studio 2017 R2 Client [43] and PyMol [34] were used to generate all the graphics.

\section{Molecular dynamics (MD) simulation}

The Ligand-ACE2-RBD complex was obtained after the targeted molecular docking. The simulation systems for ACE2-RBD complex without or with the Withanone were prepared using the VMD software (Humphrey et al., 1996). Ligand parameterization was done with CHARMM-GUI web interface (http://www.charmm-gui.org) [44]. MD simulation was performed with CHARMM36 force field using the NAMD package [45]. The protein complex without or with Withanone was solvated with TIP3P water molecules $10 \AA$ from the protein. The systems were ionized and neutralized with $100 \mathrm{mM}$ of $\mathrm{NaCl}$. The systems contained 67890 and 67851 water molecules in the protein complex without and with the Withanone, respectively. NPT ensemble was used with periodic boundary conditions. Pressure was fixed at $1 \mathrm{~atm}$, while the temperature was $310 \mathrm{~K}$. The particle-mesh Ewald method was used to evaluate the Coulomb interactions. $2 \mathrm{fs}$ of time step was used in all MD simulations. Initially, water was equilibrated for $200 \mathrm{ps}$ at $310 \mathrm{~K}$ after fixing the protein and energy minimization of 1000 steps. 1000 steps of energy minimization of the whole system were performed, and further equilibration for $400 \mathrm{ps}$ at $310 \mathrm{~K}$ after releasing the protein was done. Production run was of $2000 \mathrm{ps}$. The trajectory data were saved at every $0.5 \mathrm{ps}$ to analyze the change in the dynamics of ACE2-RBD binding interface. The results for flexibility were analyzed by plotting the non-H atoms RMSD values against the 1000 conformations (stride 4). Trajectory clustering was performed by UCSF Chimera-1.13.1 [35], using the step size of 1 and default parameters.

\section{Salt bridge analysis}

Salt bridge was defined at a cut off distance of $3.2 \AA$ of O-N atoms of the oppositely charged amino acid side chains using VMD [46]. Electrostatic free energies up on salt bridge formation were computed in initial and final trajectories. All the computations were performed according to the protocol of Hendsch and Tidor [47], with slight modifications. Briefly, it was calculated relative to a mutation of its salt-bridging side-chains to their hydrophobic isosteres; they are identical with the charged residue side-chains, with the exception that their partial atomic charges were set to zero. The protonation states of all the charged residues were assigned at $\mathrm{pH} 7.4$ using ProteinPrepare module in PlayMolecule (https://www.playmolecule.org). Continuum electrostatic calculations were performed with the DelPhi v8.4.3 [48]. The PARSE partial atomic charges and atomic radii [49] were used. The solvent probe radius used was $1.4 \AA$. The dielectric constants of the protein and the solvent were 4.0 and 80.0 respectively, and the ionic strength was $0.145 \mathrm{M}$. The Poisson-Boltzmann equation was solved using the iterative finite difference method, initially mapping the molecule on a 3D grid with a grid size of 165 and scale 1 , and then focusing with a grid size of 65 and scale 4 . DelPhi gives the energy values in units of $k T$, where $k$ is the Boltzmann constant and $\mathrm{T}$ is absolute temperature, and the values were multiplied by 0.5922 to obtain the results in $\mathrm{kcal} / \mathrm{mol}$ at room temperature $\left(25^{\circ} \mathrm{C}\right)$.

\section{ACE2-RBD complex' binding energy calculation}


Quantification of electrostatic interactions is important to study protein-protein interactions in biomolecular systems. We solved the Poisson-Boltzmann equation (PBE) in the implicit solvent model [50] to study the interaction between the receptor ACE2 and the viral RBD. The electrostatic component of $\Delta \Delta \mathrm{G}, \Delta \Delta \mathrm{G}_{\text {el }}$ can be quantified by solving the Poisson-Boltzmann equation (PBE) with the assumption that there are no conformational changes upon binding, and the equation can be presented as [51]:

$\Delta \Delta \mathrm{G}_{\mathrm{el}}=\Delta \Delta \mathrm{G}_{\mathrm{el} \_ \text {solv }}+\Delta \Delta \mathrm{G}_{\mathrm{el} \_ \text {coul }}$

$=\left[\left(\Delta \Delta \mathrm{G}_{\text {el_solv }}\right)_{\mathrm{C}}-\left(\Delta \Delta \mathrm{G}_{\text {el_solv }}\right)_{\mathrm{A}}-\left(\Delta \Delta \mathrm{G}_{\text {el_solv }}\right)_{\mathrm{B}}\right]+\left[\left(\Delta \Delta \mathrm{G}_{\text {el_coul }}\right)_{\mathrm{C}}-\left(\Delta \Delta \mathrm{G}_{\text {el_coul }}\right)_{\mathrm{A}}-\left(\Delta \Delta \mathrm{G}_{\text {el_coul }}\right)_{B}\right]$

Where, $\left(\Delta \Delta \mathrm{G}_{\text {el_solv }}\right)_{\mathrm{C}}$ is the electrostatic solvation free energy of the complex, and $\left(\Delta \Delta \mathrm{G}_{\text {el_solv }}\right)_{\mathrm{A}}$ and $\left(\Delta \Delta \mathrm{G}_{\text {el_solv }}\right)_{B}$ correspond to that of the binding partners. Similarly, $\left(\Delta \Delta \mathrm{G}_{\text {el_coul }}\right)_{C},\left(\Delta \Delta \mathrm{G}_{\text {el_coul }}\right)_{A}$ and $\left(\Delta \Delta \mathrm{G}_{\text {el_coul }}\right)_{\mathrm{B}}$ represent the electrostatic coulombic binding free energy of the complex, and the binding partners.

We used DelPhi v8.4.3 [48] to calculate the electrostatic component of the binding free energies in the final trajectory of the MD simulation without or with the Withanone, and in the trajectories obtained after clustering. The above term, $\Delta \Delta \mathrm{G}_{\mathrm{el}}$ can be obtained by subtracting the all energy but grid energy of the binding partners from that of the complex. Grid size of 165 , scale 1 and ionic strength of $0.000 \mathrm{M}$ were used for the calculation. Protein and solvent dielectric constants were 4 and 80.

\section{Results}

\section{Molecular docking}

The model of protein complex was built by SWISS-MODEL and the energy was minimized. The stereochemical quality was checked and confirmed (refer to Supporting Information1 for the details). Withanolides present in roots and leaves of W. Somnifera were docked against ACE2-RBD complex. The phytocompound which bound to the interface was subjected to targeted/focused docking. The phytocompounds were bound at the ACE2-RBD complex tightly (see Table 1 for Vina score). Of these compounds docked, only the Withanone bound at the interface of the receptor and RBD (Fig. 2). Therefore, Withanone was analyzed further to study its role in blocking or weakening the interactions between the ACE2 receptor and RBD. On targeted docking of the Withanone, it was found to be wellbound at the ACE2-RBD interface by two H-bonds (Tyr16 of ACE2 and Tyr175 of RBD to Withanone), alkyl and van der waals interactions (Fig. 2). These Tyrosines were mutated by alanine, and re-docked with Withanone.

Table 1: AutoDock Vina score of phytocompounds from

W. somnifera docked against ACE2-RBD complex. 


\begin{tabular}{|ll|}
\hline $\begin{array}{l}\text { W. somnifera } \\
\text { Phytocompounds }\end{array}$ & $\begin{array}{l}\text { Binding Energy } \\
\text { (kcal/mol) }\end{array}$ \\
\hline Withanone & -9.4 \\
\hline Withanolide A & -9.6 \\
\hline Withanolide B & -9.4 \\
\hline Withaferin A & -9.1 \\
\hline
\end{tabular}

\section{Molecular dynamics (MD) simulation}

\section{Interaction analysis}

The RMSD of the simulated molecule (Withanone) was $5.08 \AA$ compared to starting position. At the end of simulation, it moved slightly towards the binding interface centre (Fig. 3A). On analysing, the ligand interaction, it was found that ACE2 Y16 H-bonding to Withanone was preserved in the simulated coordinates, whereas RBD Y175 forms Carbon H-bond to Withanone. Additionally, there is formation of three more H-bonds (ACE2 N15, ACE2 Q19 and RBD R78 to Withanone) in the simulated ligand-ACE2-RBD complex (Fig. 3B).

\section{Salt bridge analysis}

We detected two inter-chain (binding interface) salt bridge interactions, Glu12 OE2- Lys87 NZ (2.75 $)$ (aa 404) and Glu20 OE2 - Arg73 NZ (2.67 A) (aa 390). Val residue (aa 404) in SARS-CoV is substituted by Lys in COVID-19 S protein RBD, and Lys (aa390) is substituted by Arg(Fig. 4). These salt bridges were also seen during the simulation. Percent occupancy of Glu12-Lys87 salt bridge was decreased to $24.3 \%$ in the simulation trajectories with the Withanone, from $47.2 \%$ present in the trajectories without the ligand. Similarly, occupancy of Glu20-Arg73 salt bridge in the trajectories with the Withanone was $80 \%$, while it was $93.8 \%$ in the trajectories without the ligand.

Energetics of the two salt bridges (E12-K87 and E20-R73) was calculated. It was $-1.36 \mathrm{kcal} / \mathrm{mol}$ and 1.53 $\mathrm{kcal} / \mathrm{mol}$ in the starting and last frame for the salt bridge E12-K87, whereas it was estimated to be -6.15 $\mathrm{kcal} / \mathrm{mol}$ and $-2.46 \mathrm{kcal} / \mathrm{mol}$ for the salt bridge E20-R73 (Figure 5).

\section{Flexibility analysis}

For local flexibility analysis, total RMSD of non-H atoms of the binding interface residues of RBD, RMSD of non-H atoms of aa 424 to 494 was determined (Fig. 6). The data shows the local RMSD changes. RMSD was decreased in the simulation of Withanone plus case, in the region of aa 424 to 494 (binding interface residues in $\mathrm{RBD}$ ). 


\section{ACE2-RBD complex' electrostatic component of binding energy calculation}

In MD simulation, total 210 and 217 clusters were obtained upon clustering analysis of the trajectories with or without Withanone, respectively. The electrostatic component of the binding free energies of ACE2-RBD complex were estimated on the last 100 ps representative trajectories of the cluster (11 trajectories, without the ligand; 10 trajectories, with the ligand) using DelPhi v8.4.3 [48], to assess the hypothesis that the proposed phyto-compound weakens the interactions between ACE2 and RBD. The $\Delta \Delta \mathrm{G}_{\mathrm{el}}$ of the complex with the ligand $(7.27 \mathrm{kcal} / \mathrm{mol})$ was decreased by $4.3 \mathrm{kcal} / \mathrm{mol}$ as compared to that without the ligand $(11.55 \mathrm{kcal} / \mathrm{mol})$, when estimated in the final trajectories, and it was decreased by $0.6 \mathrm{kcal} / \mathrm{mol}$ when estimated on the last $100 \mathrm{ps}$ representative trajectories $(8.89$ and $8.33 \mathrm{kcal} / \mathrm{mol}$ in the trajectories without or with the ligand, respectively) (Figure. 7). This indicates that the binding of Withanone at the interface of the ACE2 and RBD weakens their interactions.

\section{Discussion}

The spike protein (S) of SARS-coronavirus (SARS-CoV) interacts to its cellular receptor, Angiotensin Converting Enzyme 2 (ACE2) via Receptor Binding Motif (RBM; aa 424-494) present in receptor binding domain (RBD). 18 residues of the receptor make contact with 14 residues of the viral spike protein, mainly by hydrophilic interactions. Tyrosine residues are present at this interface, in RBM [23]. Besides these, Prabakaran et al. [18] also noted the importance of tyrosines while studying the binding interface of the RBD and neutralizing antibody. This tyrosine was also noted by Prabakaran et al. [18] as an important residue in making interaction to neutralizing antibody. We also observed important role of tyrosines; the phytocompound, 'Withanone', was well-bound at the ACE2-RBD interface by two H-bonds (Tyr16 of ACE2 and Tyr175 of RBD to Withanone) (Table 1, Fig. 2), and upon mutation of RBD Tyr175 to Ala, the phytocompound preferred different location.

MD simulation of ACE2-RBD complex was performed using NAMD, with or without the ligand molecule (Withanone). The Withanone moved slightly towards the centre of the binding interfacewith a formation of three new H-bonds (ACE2 N15, ACE2 Q19 and RBD R78 to Withanone) (Figs. 3A and 3B). Further, we explored ionic interactions at the binding interface of the modeled ACE2 receptor and RBD of 2019nCoV.Two inter-chain (binding interface) salt bridge interactions were detected: Glu12 OE2- Lys87 NZ (2.75 $\AA$ ) (aa 404) and Glu20 OE2 - Arg73 NZ (2.67 Å) (aa 390) (Fig. 4A). These salt bridge interactions play an important role in stabilization of the ACE2 receptor and RBD complex. Val residue (aa 404) in SARS-CoV is substituted by Lys in COVID-19 S protein RBD, and Lys (aa390) is substituted by Arg. Overall, the increase in salt bridge number in the receptor-RBD binding interface makes the complex more stabilized in COVID-19 as compared to SARS-CoV (Fig. 4A). Percent occupancies of the salt bridges were decreased in the simulation trajectories with the Withanone, as compared to the trajectories without the Withanone. Overall, there were observed effects of the ligand incorporation in these salt bridges 
occupancies. Longer simulation is needed to observe whether these salt bridges are completely broken by incorporating the Withanone.

Protein surfaces have many hydrophilic residues, and salt bridges present in the surface play an important role in protein-protein association or binding [52]. Hence the protein interface (binding interface) is generally more hydrophilic than the protein interiors. Xu et al. [53] showed that electrostatic interactions play an important role in protein binding than in folding. Hence the interfacial salt bridges, which are the major contributors to the electrostatic interactions between proteins, get central role in binding events. Generally, the structures of the proteins do not change significantly upon complex formation, but some conformational rearrangements are observed, and most of these are in side chain movements [54]. Geometrical complementarity and stability in energetic are the two factors to occur for protein binding, and the hydrophobic effect, hydrogen bonds and salt bridges are the key players in energetic. A salt bridge can provide favorable free energy to the binding [53], on the other hand, an isolated charge without forming a salt bridge, when buried in the protein interface, could substantially destabilize binding, due to the to the desolvation cost. We calculated energetics of the two salt bridges (E12-K87 and E20-R73) at the interface of ACE2 and RBD. Both the salt bridges were stabilizing initially $(-1.36 \mathrm{kcal} / \mathrm{mol}$ and $-6.15 \mathrm{kcal} / \mathrm{mol}$, respectively), but turned into destabilizing (salt bridge E12-K87, $1.53 \mathrm{kcal} / \mathrm{mol}$ ) or less stabilizing (salt bridge E20-R73, $-2.46 \mathrm{kcal} / \mathrm{mol}$ ) as seen in last frame of the MD simulation (Fig. 5). These results clearly indicated that the incorporation of the Withanone had pronounced effect on the salt bridges and on protein complex stability.

In the current work, we have presented the results of a MD simulation of the ACE2-RBD complex with and without Withanone. The data shows the local RMSD changes, a measure of flexibility, of non-H atoms of the binding interface residues of RBD (aa 424 to 494) (Fig. 6). RMSD was decreased in the simulation of Withanone plus case, in the region of aa424 to 494. It may be due to the involvement of these contact residues in electrostatic interactions with the ligand.

The electrostatic component of the binding free energies of ACE2-RBD complex were estimated on 11 trajectories (simulated without the ligand) and 10 trajectories (simulated with the ligand), to assess the hypothesis that the proposed phytocompound weakens the interactions between ACE2 and RBD. $\triangle \triangle G_{\text {el }}$ was decreased by $0.6 \mathrm{kcal} / \mathrm{mol}$ in the simulation trajectories with the Withanone, compared to the trajectories simulated without the Withanone $(8.89$ and $8.33 \mathrm{kcal} / \mathrm{mol}$ in the trajectories without or with the ligand, respectively) (Figure. 7). Whereas, the $\Delta \Delta \mathrm{G}_{\mathrm{el}}$ of the complex with the ligand $(7.27 \mathrm{kcal} / \mathrm{mol})$ was decreased by $4.3 \mathrm{kcal} / \mathrm{mol}$ as compared to that without the ligand $(11.55 \mathrm{kcal} / \mathrm{mol})$. Such a decrease in electrostatic component of binding free energy clearly indicates that the binding of Withanone at the interface of the ACE2 and RBD weakens their interactions.

\section{Conclusions}

We have developed a strategy that may be utilized to block or weaken the viral infections like COVID-19, via disruption of the electrostatic interaction of the interacting viral protein with the ACE2 receptor. In this 
regard, phytochemicals present in Indian medicinal herb Withania somnifera(Ashwagandha) were screened by molecular docking, and simulated with the selected phytocompound, Withanone. Proteinprotein interactions were studied by calculating the electrostatic component of binding free energy between the viral RBD and its ACE2 receptor in the presence or absence of Withanone. Salt bridges' occupancies also decreased in the trajectories simulated with the Withanone, along with the concurrent decrease in the electrostatic components of binding free energy. We also observed the stabilizing salt bridges turning into destabilizing, at the end of simulation. This study highlights the importance of natural origin phytochemicals in controlling COVID-19 entry into host cells, and provides an attractive and alternative means for the management of COVID-19 infection. W. somnifera could well be the first choice of medicinal herbs in these directions, to control the COVID-19 infectivity.

\section{List Of Abbreviations}

COVID-19, Coronavirus disease 2019; S-protein, Spike protein; ACE2, Angiotensin converting enzyme-2; RBD, Receptor binding domain; 2019-nCoV, Novel Coronavirus 2019; MD, Molecular dynamics; CCDCP, Chinese Center for Disease Control and Prevention; SARS-CoV, Severe Acute Respiratory SyndromeCoronavirus; MERS-CoV, Middle East Respiratory Syndrome-Coronavirus; nAbs, Neutralizing antibodies; Nterminal, Amino terminal; C-terminal, Carboxy terminal; W. somnifera, Withaniasomnifera; PBE, PoissonBoltzmann equation; ADT4, Autodock Tools 4; NAMD, Nanosclae molecular dynamics; VMD, Visual molecular dynamics; RMSD, Root mean square deviation; E20-R73, Glu20-Arg73; E12-K87, Glu12-Lys87; aa491, Amino acid 491; RBM, Receptor binding motif

\section{Declarations}

Author Contributions: Conceptualization and resource generation, A.B.; Formal analysis, S.P., A.V.; Investigation, S.P.; Methodology, S.P.; Software, S.P.; Supervision, A.V.; Writing-original draft, S.P., J. S.; Writing-review \& editing, A.V.

Funding: This research received funding from Patanjali Research Foundation Trust, Haridwar, India.

Acknowledgments: The authors are indebted to Param Shradhey Swami Ramdev Ji for his financial and institutional supports to accomplish this research work. We extend our gratitude to Ms. Priyanka Kandpal, Mr. Tarun Rajput, Mr. Gagan Kumar, and Mr. Lalit Mohan for their swift administrative supports.

Conflicts of Interest: The authors declare no conflict of interest.

Availability of data and materials: All the data generated or analyzed during this study are included in this manuscript.

Ethics approval and consent to participate: Not applicable.

Consent for publication: Not applicable. 


\section{References}

1. Lu H, Stratton CW, Tang YW. Outbreak of Pneumonia of Unknown Etiology in Wuhan China: the Mystery and the Miracle. Journal of Medical Virology 2020.

2. Zhu N, Zhang D, Wang W, Li X, Yang B, Song J, Zhao X, Huang B, Shi W, Lu R, Niu P. A novel coronavirus from patients with pneumonia in China, 2019. New England Journal of Medicine. 2020 Jan 24.

3. Kahn N. New virus discovered by Chinese scientists investigating pneumonia outbreak. Wall Street Journal. 2020.

4. Kruse RL. Therapeutic strategies in an outbreak scenario to treat the novel coronavirus originating in Wuhan, China. F1000Research. 2020;9.

5. Gralinski LE, Menachery VD. Return of the Coronavirus: 2019-nCoV. Viruses. 2020 Feb;12(2):135.

6. RichmanDD, Whitley RJ, HaydenFG. Clinical virology2016, (4th edn.), ASM Press, Washington

7. Zhang J, Zhou L, Yang Y, Peng W, Wang W, Chen X. Therapeutic and triage strategies for 2019 novel coronavirus disease in fever clinics. The Lancet Respiratory Medicine. 2020 Feb 13.

8. Chan JF, Yuan S, Kok KH, To KK, Chu H, Yang J, Xing F, Liu J, Yip CC, Poon RW, Tsoi HW. A familial cluster of pneumonia associated with the 2019 novel coronavirus indicating person-to-person transmission: a study of a family cluster. The Lancet. 2020 Feb 15;395(10223):514-23.

9. Zeng ZQ, Chen DH, Tan WP, Qiu SY, Xu D, Liang HX, Chen MX, Li X, Lin ZS, Liu WK, Zhou R. Epidemiology and clinical characteristics of human coronaviruses OC43, 229E, NL63, and HKU1: a study of hospitalized children with acute respiratory tract infection in Guangzhou, China. European Journal of Clinical Microbiology \& Infectious Diseases. 2018 Feb 1;37(2):363-9.

10. Ksiazek TG, Erdman D, Goldsmith CS, Zaki SR, Peret T, Emery S, Tong S, Urbani C, Comer JA, Lim W, Rollin PE. A novel coronavirus associated with severe acute respiratory syndrome. New England journal of medicine. 2003 May 15;348(20):1953-66.

11. Stadler K, Masignani V, Eickmann M, Becker S, Abrignani S, Klenk HD, Rappuoli R. SARS-beginning to understand a new virus. Nature Reviews Microbiology. 2003 Dec;1(3):209-18.

12. Zaki AM, Van Boheemen S, Bestebroer TM, Osterhaus AD, Fouchier RA. Isolation of a novel coronavirus from a man with pneumonia in Saudi Arabia. New England Journal of Medicine. 2012 Nov 8;367(19):1814-20.

13. Huang C, Wang Y, Li X, Ren L, Zhao J, Hu Y, Zhang L, Fan G, Xu J, Gu X, Cheng Z. Clinical features of patients infected with 2019 novel coronavirus in Wuhan, China. The Lancet. 2020 Feb 15;395(10223):497-506.

14. Hui DS, I Azhar E, Madani TA, Ntoumi F, Kock R, Dar O, Ippolito G, Mchugh TD, Memish ZA, Drosten C, Zumla A. The continuing 2019-nCoV epidemic threat of novel coronaviruses to global health-The latest 2019 novel coronavirus outbreak in Wuhan, China. International Journal of Infectious Diseases. 2020;91:264-6. 
15. Bupp K, Roth MJ. Alteration and Analyses of Viral Entry with Library-Derived Peptides. Advances in virus research. 2005 Jan 1;65:147-72.

16. Douglas JL, Panis ML, Ho E, Lin KY, Krawczyk SH, Grant DM, Cai R, Swaminathan S, Cihlar T. Inhibition of respiratory syncytial virus fusion by the small molecule VP-14637 via specific interactions with F protein. Journal of virology. 2003 May 1;77(9):5054-64.

17. Razinkov V, Gazumyan A, Nikitenko A, Ellestad G, Krishnamurthy G. RFI-641 inhibits entry of respiratory syncytial virus via interactions with fusion protein. Chemistry \& biology. $2001 \mathrm{Jul}$ $1 ; 8(7): 645-59$.

18. Prabakaran P, Gan J, Feng Y, Zhu Z, Choudhry V, Xiao X, Ji X, Dimitrov DS. Structure of severe acute respiratory syndrome coronavirus receptor-binding domain complexed with neutralizing antibody. Journal of Biological Chemistry. 2006 Jun 9;281(23):15829- 36.

19. Adedeji AO, Severson W, Jonsson C, Singh K, Weiss SR, Sarafianos SG. Novel inhibitors of severe acute respiratory syndrome coronavirus entry that act by three distinct mechanisms. Journal of virology. 2013 Jul 15;87(14):8017-28.

20. Millet JK, Whittaker GR. Host cell proteases: Critical determinants of coronavirus tropism and pathogenesis. Virus research. 2015 Apr 16;202:120-34.

21. Bosch BJ, Bartelink W, Rottier PJ. Cathepsin L functionally cleaves the severe acute respiratory syndrome coronavirus class I fusion protein upstream of rather than adjacent to the fusion peptide. Journal of virology. 2008 Sep 1;82(17):8887-90.

22. Matsuyama S, Nagata N, Shirato K, Kawase M, Takeda M, Taguchi F. Efficient activation of the severe acute respiratory syndrome coronavirus spike protein by the transmembrane protease TMPRSS2. Journal of virology. 2010 Dec 15;84(24):12658-64.

23. Li F, Li W, Farzan M, Harrison SC. Structure of SARS coronavirus spike receptor- binding domain complexed with receptor. Science. 2005 Sep 16;309(5742):1864-8.

24. Wan Y, Shang J, Graham R, Baric RS, Li F. Receptor recognition by novel coronavirus from Wuhan: An analysis based on decade-long structural studies of SARS. Journal of virology. 2020 Jan 29.

25. Tian X, Li C, Huang A, Xia S, Lu S, Shi Z, Lu L, Jiang S, Yang Z, Wu Y, Ying T. Potent binding of 2019 novel coronavirus spike protein by a SARS coronavirus-specific human monoclonal antibody. Emerging Microbes \& Infections. 2020 Jan 1;9(1):382-5.

26. Dau B, Holodniy M. Novel Targets for antiretroviral therapy. Drugs. 2009 Jan 1;69(1):31-50.

27. Anand K, Ziebuhr J, Wadhwani P, Mesters JR, Hilgenfeld R. Coronavirus main proteinase (3CLpro) structure: basis for design of anti-SARS drugs. Science. 2003 Jun 13;300(5626):1763-7.

28. Sangwan RS, Chaurasiya ND, Misra LN, Lal P, Uniyal GC, Sharma R, Sangwan NS, Suri KA, Qazi GN, Tuli R. Phytochemical variability in commercial herbal products and preparations of Withaniasomnifera (Ashwagandha). Current Science. 2004 Feb 10:461- 5.

29. Kambizi LG, Goosen BM, Taylor MB, Afolayan AJ. Anti-viral effects of aqueous extracts of Aloe ferox and Withaniasomnifera on herpes simplex virus type 1 in cell culture. South African Journal of Science. 2007 Oct;103(9-10):359-60. 
30. Cai Z, Zhang G, Tang B, Liu Y, Fu X, Zhang X. Promising anti-influenza properties of active constituent of Withaniasomnifera ayurvedic herb in targeting neuraminidase of

H1N1 influenza: computational study. Cell biochemistry and biophysics. $2015 \mathrm{Jul}$ 1;72(3):727-39.

31. Chaurasiya ND, Uniyal GC, Lal P, Misra L, Sangwan NS, Tuli R, Sangwan RS. Analysis of withanolides in root and leaf of Withaniasomnifera by HPLC with photodiode array and evaporative light scattering detection. Phytochemical Analysis: An International Journal of Plant Chemical and Biochemical Techniques. 2008 Mar;19(2):148-54.

32. Zhang C, Zheng W, Huang X, Bell EW, Zhou X, Zhang Y. Protein structure and sequence re-analysis of 2019-nCoV genome does not indicate snakes as its intermediate host or the unique similarity between its spike protein insertions and HIV-1. arXiv preprint arXiv:2002.03173. 2020 Feb 8.

33. Šali A, Blundell TL. Comparative protein modelling by satisfaction of spatial restraints. Journal of molecular biology. 1993 Dec 5;234(3):779-815.

34. DeLano WL. Pymol: An open-source molecular graphics tool. CCP4 Newsletter on protein crystallography. 2002 Mar;40(1):82-92.35. Pettersen EF, Goddard TD, Huang CC, Couch GS, Greenblatt DM, Meng EC, Ferrin TE. UCSF Chimera-a visualization system for exploratory research and analysis. Journal of computational chemistry. 2004 Oct;25(13):1605-12.

35. Lüthy R, Bowie JU, Eisenberg D. Assessment of protein models with three-dimensional profiles. Nature. 1992 Mar;356(6364):83-5.37. Colovos C, Yeates TO. Verification of protein structures: patterns of nonbonded atomic interactions. Protein science. 1993 Sep;2(9):1511-9.

36. Laskowski RA, MacArthur MW, Moss DS, Thornton JM. PROCHECK: a program to check the stereochemical quality of protein structures. Journal of applied crystallography. 1993 Apr 1;26(2):283-91.

37. Lovell SC, Davis IW, Arendall WB, de Bakker PI, Word JM, Prisant MG, Richardson JS, Richardson DC. Structure validation by Calpha geometry: phi, psi and Cbeta deviation. Proteins 50, 437e450.

38. O'Boyle NM, Banck M, James CA, Morley C, Vandermeersch T, Hutchison GR. Open Babel: An open chemical toolbox. Journal of cheminformatics. 2011 Dec;3(1):33.

39. Trott O, Olson AJ. AutoDock Vina: improving the speed and accuracy of docking with a new scoring function, efficient optimization, and multithreading. Journal of computational chemistry. 2010 Jan 30;31(2):455-61.

40. Morris GM, Huey R, Lindstrom W, Sanner MF, Belew RK, Goodsell DS, Olson AJ. AutoDock4 and AutoDockTools4: Automated docking with selective receptor flexibility. Journal of computational chemistry. 2009 Dec;30(16):2785-91.

41. Dassault Systèmes BIOVIA, Discovery Studio 2017 R2 Client, Release 2017, San Diego: Dassault Systèmes, 2017.

42. Jo S, Kim T, Iyer VG, Im W. CHARMM-GUl: a web-based graphical user interface for CHARMM. Journal of computational chemistry. 2008 Aug;29(11):1859-65.

43. Phillips JC, Braun R, Wang W, Gumbart J, Tajkhorshid E, Villa E, Chipot C, Skeel RD, Kale L, Schulten K. Scalable molecular dynamics with NAMD. Journal of computational chemistry. 2005 
Dec;26(16):1781-802.

44. Humphrey W, Dalke A, Schulten K. VMD: visual molecular dynamics. Journal of molecular graphics. 1996 Feb 1;14(1):33-8.

45. Hendsch ZS, Tidor B. Do salt bridges stabilize proteins? A continuum electrostatic analysis. Protein Science. 1994 Feb;3(2):211-26.

46. Li L, Li C, Sarkar S, Zhang J, Witham S, Zhang Z, Wang L, Smith N, Petukh M, Alexov E. DelPhi: a comprehensive suite for DelPhi software and associated resources. BMC biophysics. 2012 Dec 1;5(1):9.

47. Sitkoff D, Sharp KA, Honig B. Accurate calculation of hydration free energies using macroscopic solvent models. The Journal of Physical Chemistry. 1994 Feb;98(7):1978- 88.

48. Fogolari F, Brigo A, Molinari $\mathrm{H}$. The Poisson-Boltzmann equation for biomolecular electrostatics: a tool for structural biology. Journal of Molecular Recognition. 2002 Nov;15(6):377-92.

49. Baker NA. Poisson-Boltzmann methods for biomolecular electrostatics. InMethods in enzymology 2004 Jan 1 (Vol. 383, pp. 94-118). Academic Press.

50. Barlow DJ, Thornton JM. The distribution of charged groups in proteins. Biopolymers: Original Research on Biomolecules. 1986 Sep;25(9):1717-33.

51. Xu D, Lin SL, Nussinov R. Protein binding versus protein folding: the role of hydrophilic bridges in protein associations. Journal of molecular biology. 1997 Jan 10;265(1):68-84.

52. Norel R, Wolfson HJ, Nussinov R. Small molecule recognition: solid angles surface representation and molecular shape complementarity. Combinatorial Chemistry and High Throughput Screening. 1999 Aug 1;2:223-36.

\section{Figures}




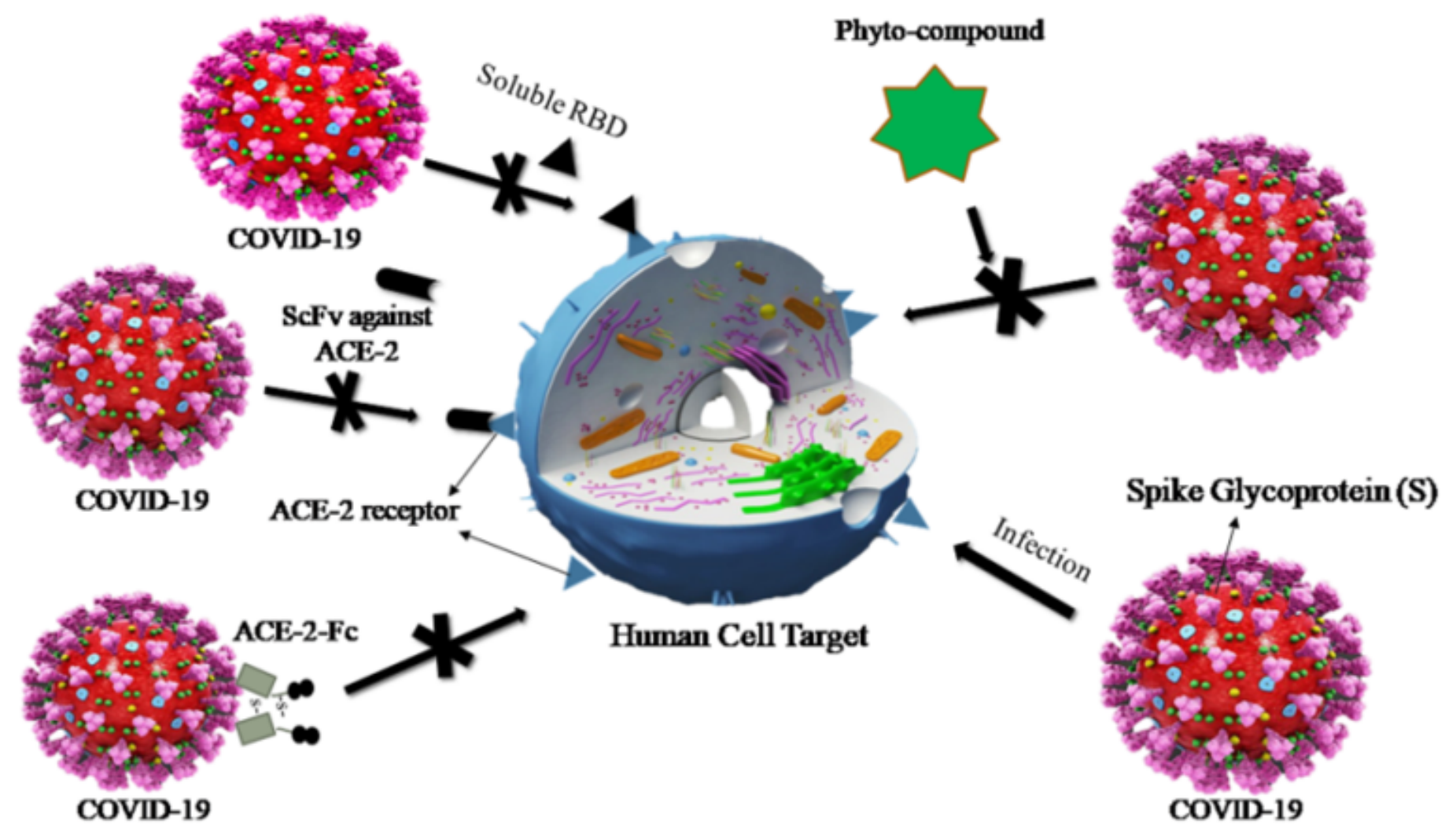

Figure 1

The possible mechanisms of therapeutic agents to block the entry of COVID-19 in to host cells. Three proposed model has been described where COVID-19 infection can be abrogated by blocking the interaction of spike protein and ACE-2. In the first model, the RBD of COVID-19 spike protein would be managed, thereby blocking ACE2 and saturating available sites. Secondly, an antibody or single-chain antibody fragment (ScFv) could be directed against ACE-2 to accomplish the same. In the third model, coronavirus virions could be directly target using the ACE-2 extracellular domain as a template to bind to virus surface protein. An Fc domain fused to ACE-2 would facilitate prolonged circulation of the biologic (ACE2-FC). Our proposed strategy is to block or weaken the interaction between RBD and ACE2 by using phytocompounds of natural origin. 


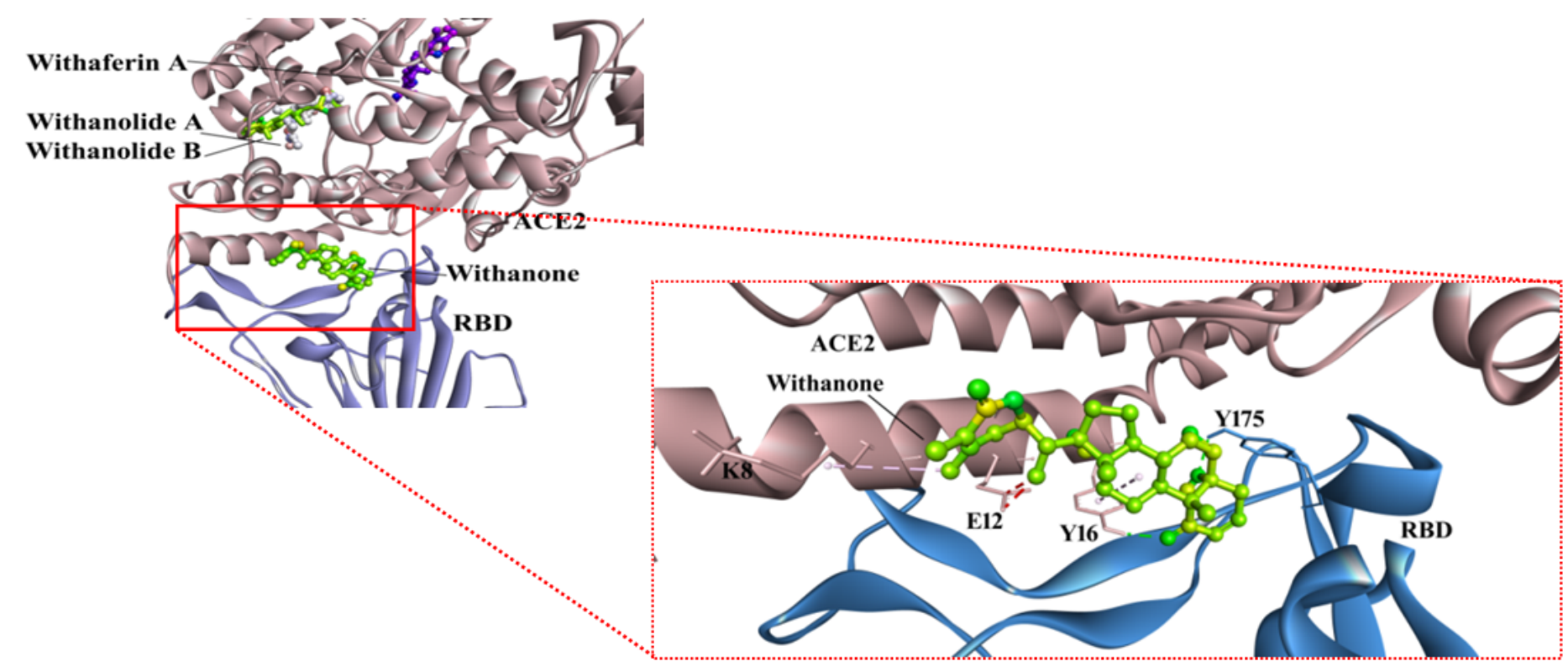

Figure 2

Binding poses of withanolides (from W. somnifera) in ACE2-RBD complex. Withanone interactions at the interface of ACE2-RBD complex has been zoomed into. Withanone is well established in the pocket by two H-bonds - Y16 of ACE2, and Y175 (aa491) of RBD, in addition to alkyl interactions. 
(A)

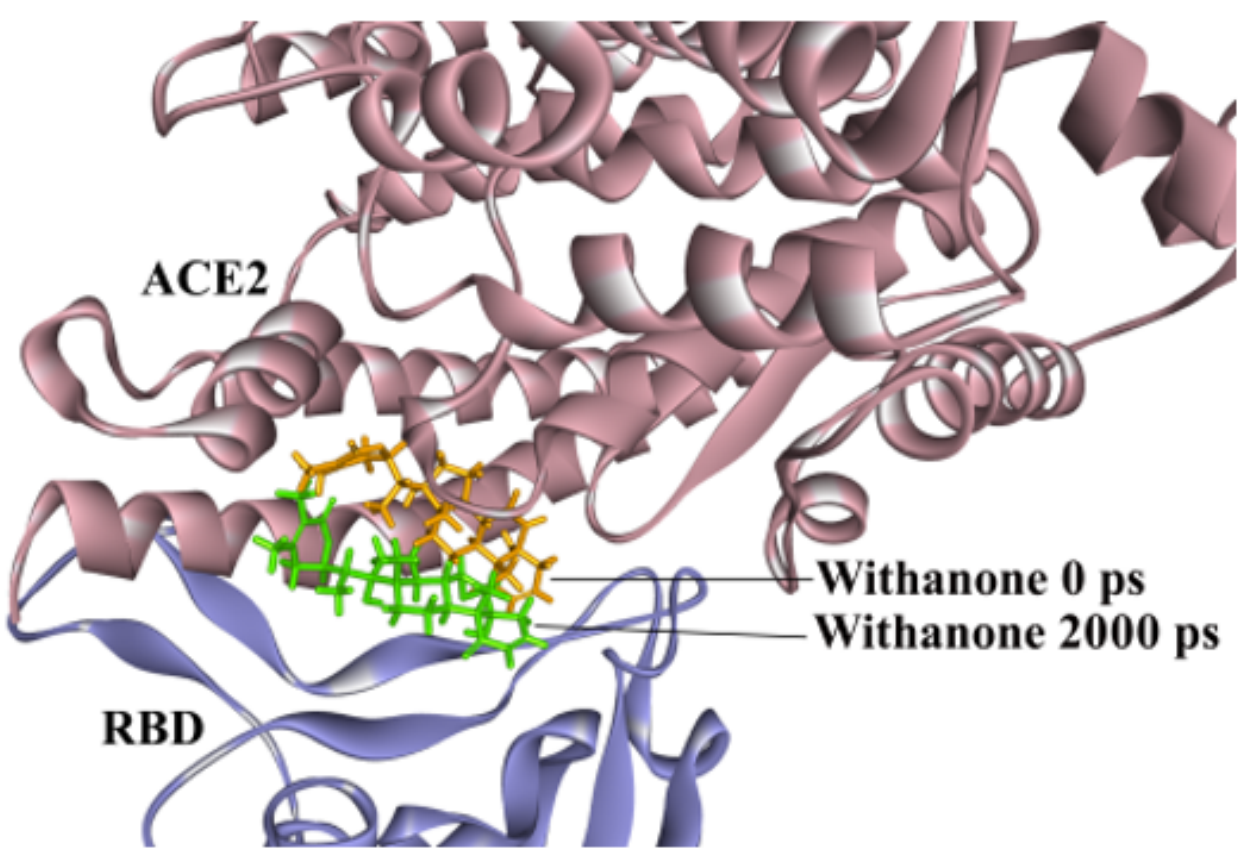

(B)

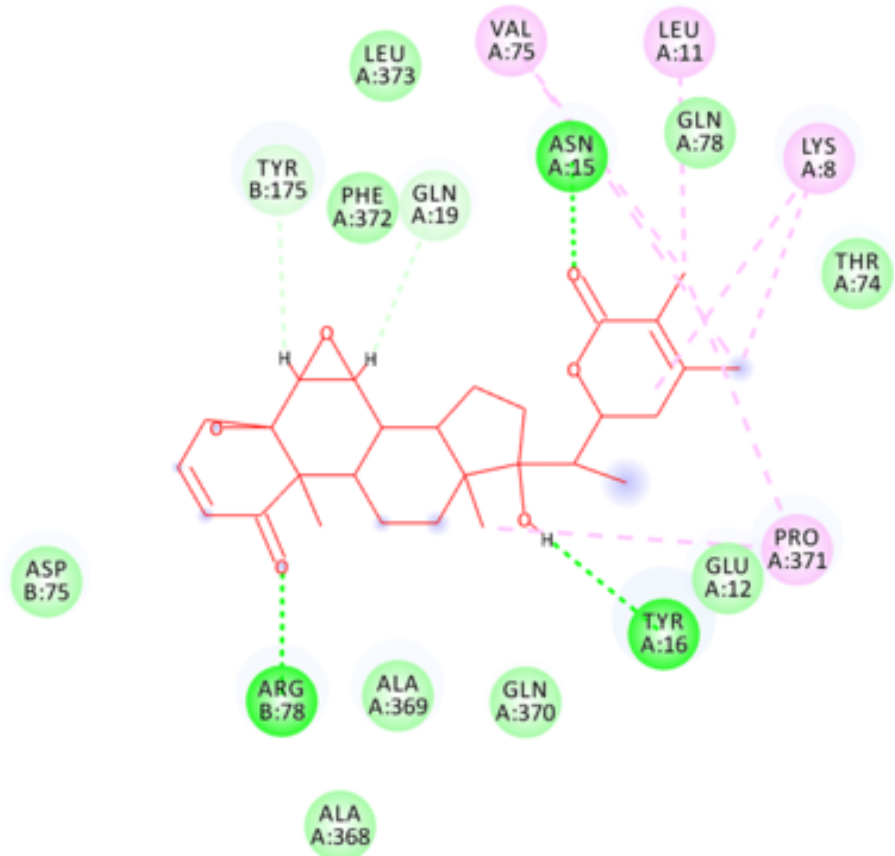

Interactions

van der Waals

Conventional Hydrogen Bond

Carbon Hydrogen Bond Alkyl

\section{Figure 3}

(A). Comparison of Withanone positions before and after MD simulation in ACE2-RBD complex. RMSD of all atoms is $5.08 \AA$. (B). Withanone' interactions within ACE2-RBD complex as seen in the final trajectory. 
(A)

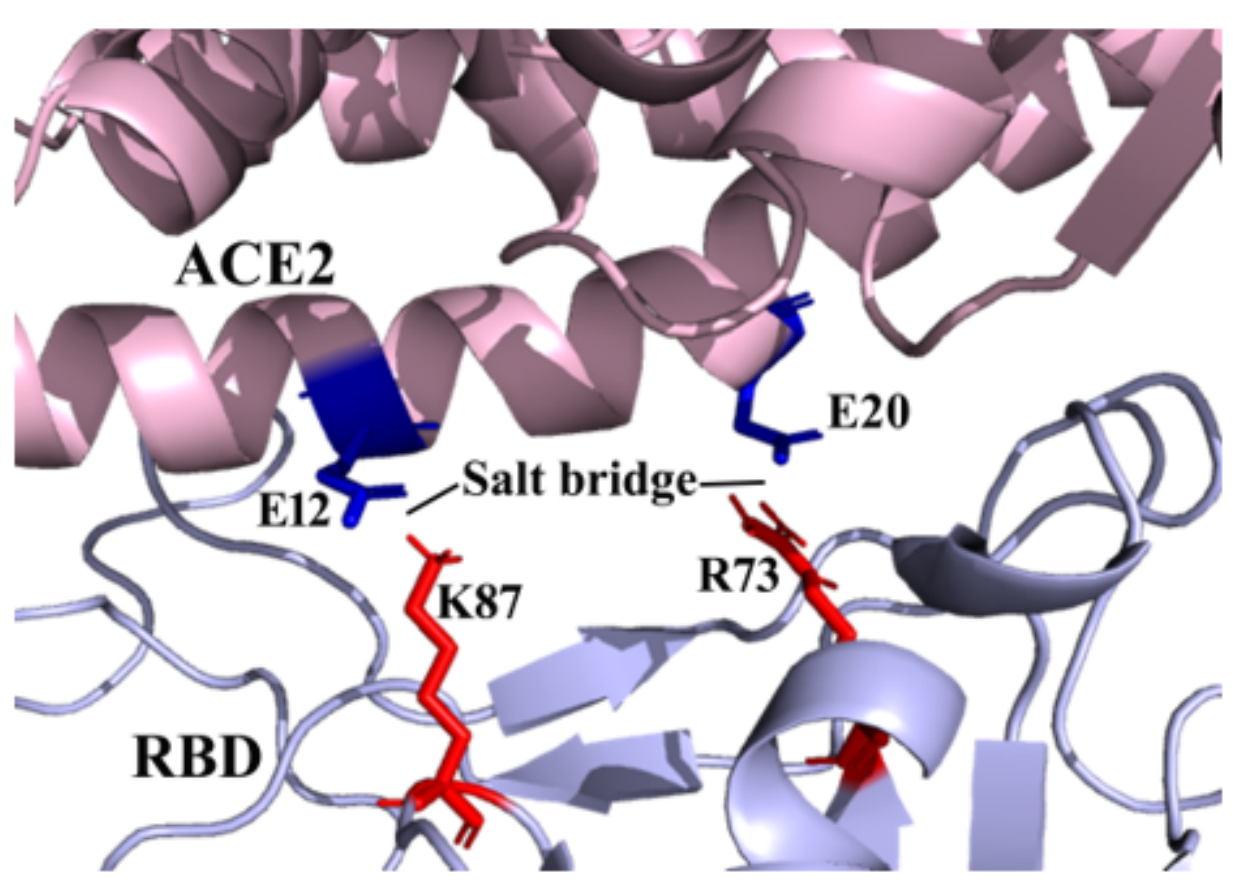

(B)

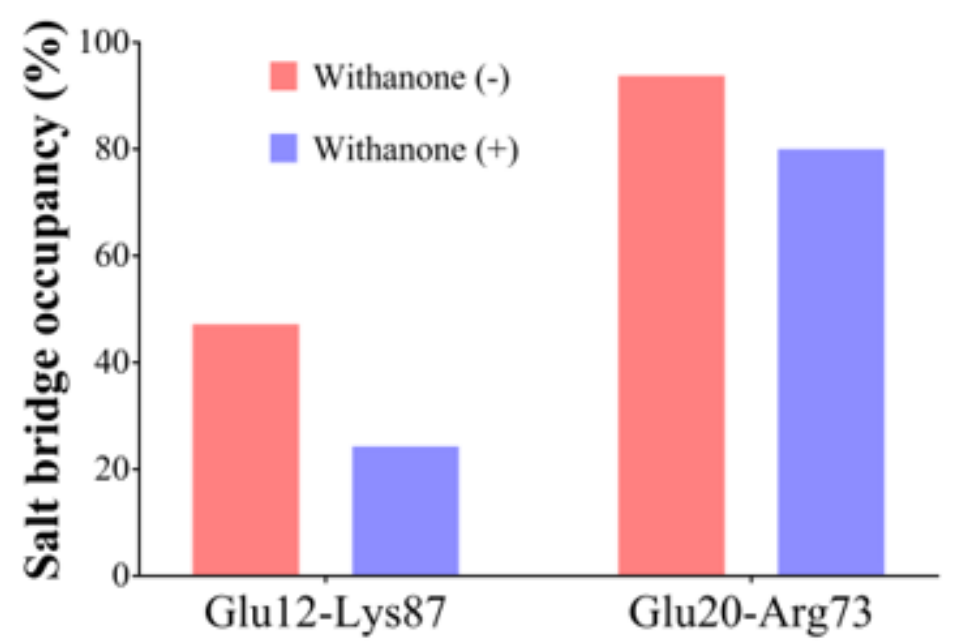

Salt bridges

Figure 4

(A). Salt bridge interactions at the binding interface of ACE2-RBD. Val87 (aa 404) in RBD of SARS-CoV is substituted by Lys in nCov, thus, introducing one extra salt bridge at the binding interface. Glu20 - Arg73 (aa 390) is preserved in both the SARS-CoV and nCoV, but there is Lys at aa 390 instead of Arg. (B).

Percent occupancy of the salt bridges modulated by Withanone incorporation as seen by analysis of the simulation trajectories. 


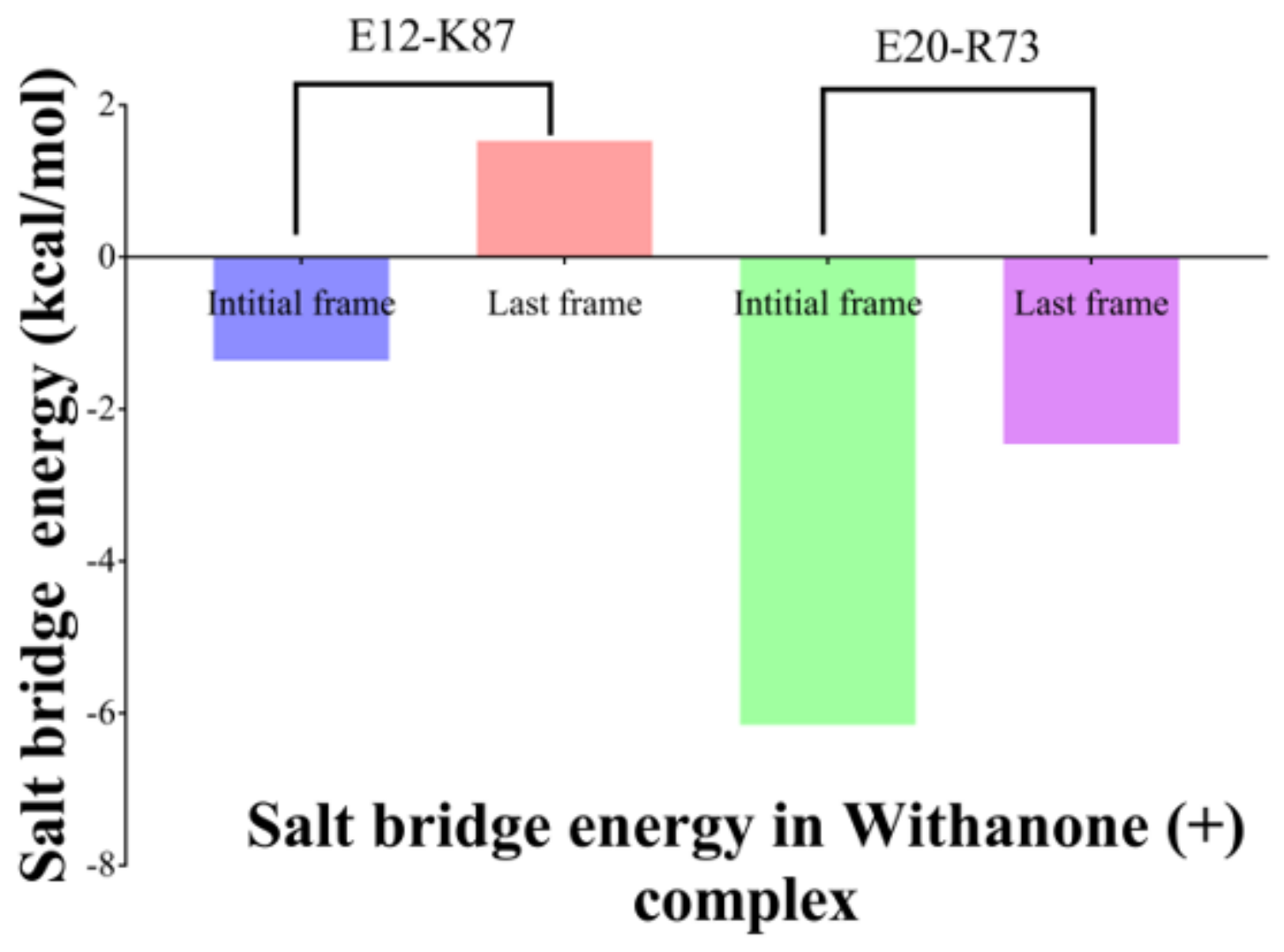

Figure 5

Electrostatic contribution upon salt bridge formation of the two salt bridges (E12-K87 and E20-R73) calculated in the initial and the final frames of simulations. E12-K87 salt bridge was energetically favorable in the first frame; gradually it got destabilized as seen in final frame. Similarly, E20-R73 salt bridge was more favorable in the initial frame, and then its extent decreased as seen in the final frame. 


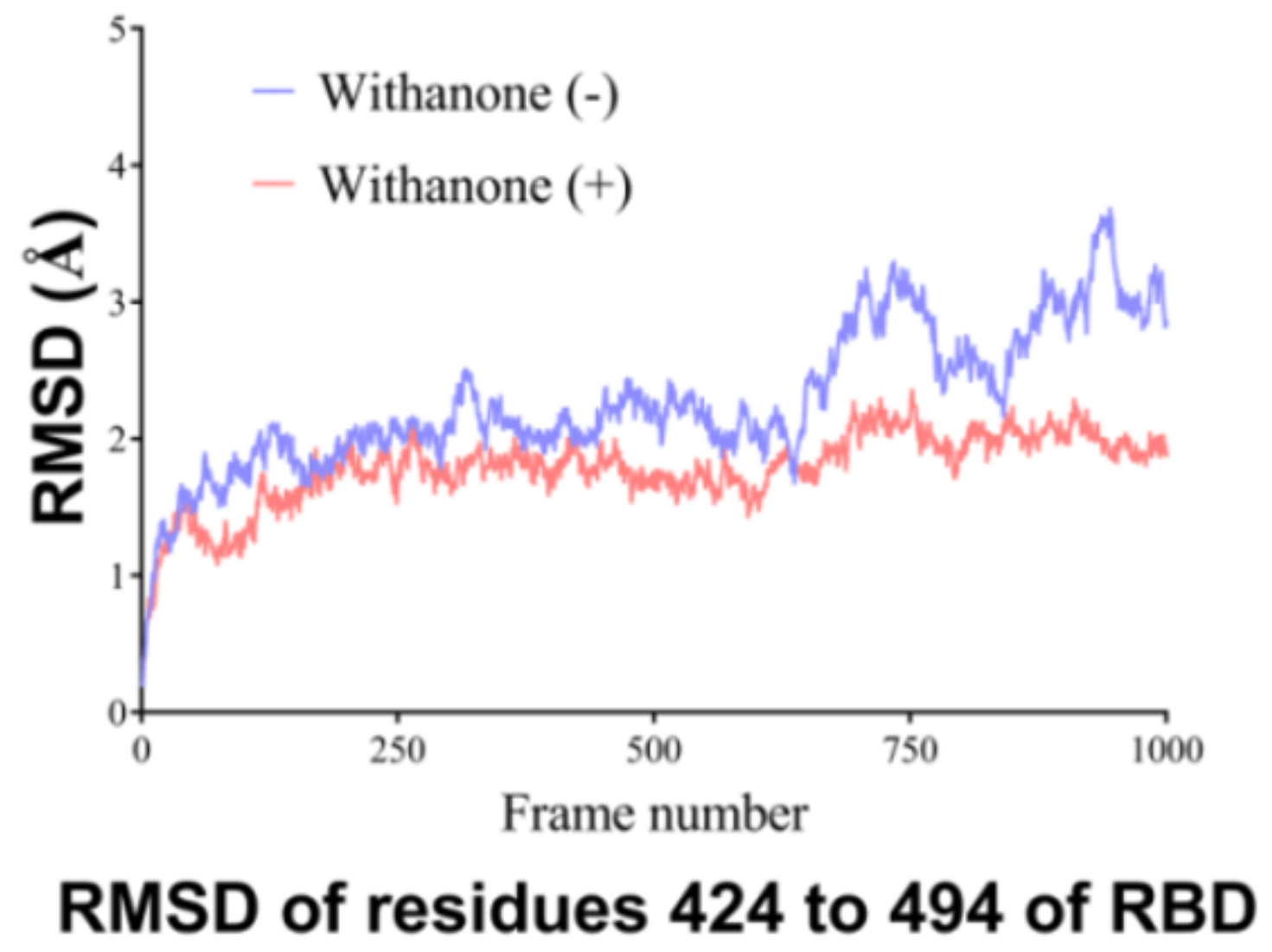

Figure 6

RMSD changes of non-H atoms of the aa424 to 494 of RBD in the ACE2-RBD complex in 2000 ps time, in the presence and absence of Withanone. 


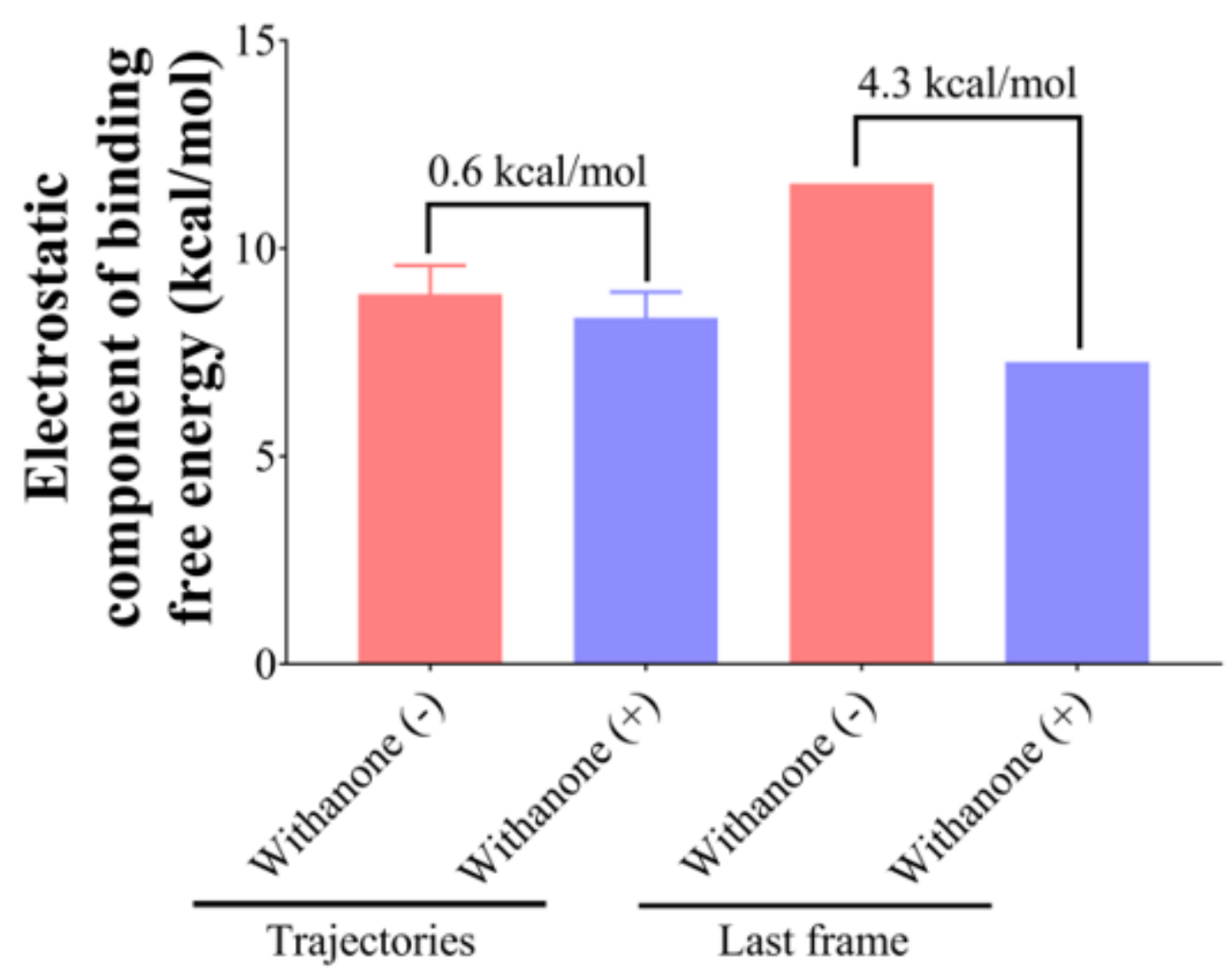

Figure 7

Comparison of electrostatic component of binding free energies in the ACE2-RBD complexes with or without the Withanone.

\section{Supplementary Files}

This is a list of supplementary files associated with this preprint. Click to download.

- Supportinglnformation1.docx 